\title{
FACTORES PSICOSOCIALES DEL TRABAJO Y SÍNDROME DE BURNOUT EN DOCENTES UNIVERSITARIOS, BLUEFIELDS, NICARAGUA
}

Eva Hodgson Suárez ${ }^{[1]}$ Arlen Soto $^{[2]}$

\section{Resumen}

El objetivo de este estudio fue determinar los factores psicosociales negativos del trabajo percibido por el trabajador, y su relación con el Síndrome de Burnout en docentes universitarios de la ciudad de Bluefields.

El tipo de estudio es cuantitativo, descriptivo, de corte transversal, realizado mediante la aplicación de la escala de factores psicosociales del trabajo académico y el Maslach Burnout Inventory, instrumentos aplicados a 109 docentes. Los resultados revelan la presencia de una alta demanda del papel académico y desarrollo de la carrera como factor negativo en el ambiente laboral de los docentes, también señala la despersonalización como la dimensión del síndrome de Burnout. Se encontró asociación entre el factor contenido y características de la tarea con la dimensión de despersonalización, así como relación entre papel académico y desarrollo de la carrera con el agotamiento emocional.

Palabras clave: Docentes universitarios, factores psicosociales, Síndrome Burnout.

\section{Summary}

The purpose of this study was to determine the negative psychosocial work factors perceived by the workers, and its relation with Burnout syndrome among university teachers in the city of Bluefields.

The study is quantitative, descriptive, cross-sectional, and was made by applying the scale of psychosocial factors of academic work and the Maslach Burnout Inventory, instruments that were applied to 109 teachers. The results reveal a high demand of the academic role and career development as a negative factor in the teachers work environment; it also refers to depersonalization as a dimension of Burnout Syndrome. Among the findings we found association between the factors related to the contents and characteristics of the task with the dimension of depersonalization, as well as

[1] IPsicóloga, Máster académica en Salud ocupacional. jcevie@gmail.com

[2] Psicóloga, Máster Profesional en Salud Ocupacional. amsv8o@yahoo.com 
relationship between the academic role and career development with emotional exhaustion.

Keywords: University teachers, psychosocial factors, Burnout Syndrome.

\section{Introducción}

Trabajar es un componente fundamental de la vida misma, por ello el ser humano dedica tiempo sustancial en el desempeño de su labor, pero así como permite ostentar a una calidad de vida satisfactoria, según las condiciones que en ella se presenten, se puede convertir en un arma que deteriore la salud de las personas.

Autores como Weber A, Jeakel R. (2000) y Preciado M, Chávez R. (2010) refieren que cuando un trabajador está expuesto a factores psicosociales negativos en su puesto laboral, que desencadenan en él alteraciones psicológicas y fisiológicas, es decir causan estrés, este es un trastorno biopsicosocial que afecta a la población mundial en diversas maneras, sus causas y efectos son variadas, pero una de las más interesantes a estudiar es el estrés laboral y su cronicidad que desenlaza en el Síndrome del Burnout.

El presente estudio, "Factores Psicosociales del trabajo y Síndrome de Burnout en docentes universitarios de la ciudad de Bluefields (Nicaragua)", señala que los componentes: papel académico, desarrollo de la carrera, contenido/características de la tarea y la baja remuneración son factores psicosociales negativos al que están expuestos los docentes en estudio. También indica la presencia de Burnout, siendo la despersonalización su dimensión más afectada. Los análisis estadísticos demuestran asociación entre la exposición a factores psicosociales negativos del trabajo y presentar Síndrome de Burnout.

Este estudio permite conocer sobre la existencia de los factores psicosociales negativos en el trabajo académico y presencia del Síndrome de Burnout, favoreciendo a que tanto docentes como autoridades universitarias diseñen y ejecuten estrategias adecuadas que permitan reducir o evitar este mal. Teóricamente se genera nueva información de otro grupo de docentes de nuestro país, lo cual dará una visión más global de la problemática en diferentes contextos académicos de Nicaragua.

\section{Revisión literaria}

\section{Factores Psicosociales del Trabajo}

La Organización Mundial del Trabajo (2008), definió los factores psicosociales del trabajo como: "las interacciones entre el contenido, organización, gestión del trabajo, condiciones ambientales con las funciones y necesidades del trabajador", estas interacciones pueden ejercer una influencia negativa sobre el individuo a través de sus 
percepciones y experiencias. Gil-Monte y Peiró (1997), identificaron que los Factores Psicosociales Negativos del trabajo, reciben también el nombre de estresores ya que generan alteraciones psicológicas y daños a la salud del individuo, incluye aspectos organizacionales, el ambiente físico de trabajo, contenido del puesto, desempeño de roles, relaciones interpersonales, desarrollo de la carrera, nuevas tecnologías, oportunidades de decisión y de realización personal, rotación de turnos y sobrecarga laboral.

Nohemí Silva (2006), identificó los factores psicosociales del trabajo académico, de igual manera, elaboró un test que permite identificar su presencia, denominado Escala de Factores Psicosociales Negativos del Trabajo Académico, a continuación una breve explicación de cada una.

a. Condiciones del lugar del trabajo: Engloba las condiciones ambientales y el diseño del puesto de trabajo.

b. Carga de trabajo: Es el conjunto de esfuerzo psicofísicos que debe ejercer el organismo durante la jornada laboral, se divide en carga física y carga mental.

c. Contenido y Características de la tarea: Considera la Complejidad de las tareas que le corresponde al individuo realizar durante la jornada laboral.

d. Exigencias Laborales: Son todos aquellos requerimientos que el docente debe desarrollar para lograr con el cumplimiento de las tareas y demandas de su puesto.

e. Papel Académico y desarrollo de la carrera: La función docente contempla varias acciones, entre ellas la preparación del plan de clases y metodología a seguir, cantidad de asignaturas a impartir, actualización en cada uno de las temáticas a desarrollar, seguimiento y tutorías a estudiantes, desarrollo de las temáticas en el aula de clases, corrección de pruebas de evaluación, participación en cursos de capacitación y programas de postgrados con el fin de asegurar un proceso de enseñanza aprendizaje optimo.

f. Interacción social. Corresponde a las relaciones interpersonales que se establecen en el puesto de trabajo, contempla también el sistema de comunicación establecido en la institución. Mientras más fluida y asertiva sea la comunicación se produce menor confusión entre roles y funciones que debe ejercer cada persona en su puesto de trabajo.

g. Remuneración y rendimiento. Este componente contempla los honorarios que reciben cada trabajador y el poder adquisitivo que tenga éste sobre bienes y servicios.

Síndrome de Burnout

El Psicoanalista Freudenberg H (1974), fue el primero en identificar el Burnout, lo describió como un trastorno psiquiátrico caracterizado por el desvanecimiento físico. En el mismo año, Moreno Jimenez B, Seminotti R y Hernández E et al (2005) 
explicaron que consiste en "una pérdida progresiva del idealismo, energía y motivos, experimentados por personas que ejercen profesiones de ayuda humanitaria, como resultado de las condiciones de trabajo". Por tal razón, también se le denomina síndrome del desgaste profesional.

Posteriormente, en 1981, Jackson C \& Maslach, diseñaron el Maslach Burnout Inventory, indicaron que el Síndrome de Burnout se produce debido a la presencia de estrés crónico en ambiente laboral, que el trabajador no ha sido capaz de resolver adecuadamente, Así mismo explican que el Burnout está configurado como un síndrome tridimensional caracterizado por:

1. El agotamiento emocional y físico: ausencia o falta de energía y entusiasmo para realizar las actividades correspondientes, predomina el cansancio, la falta de interés, poca creatividad, baja voluntad y un sentimiento de exhaustividad mental.

2. La despersonalización o deshumanización: Consiste en la pérdida de la empatía, trata a los clientes, compañeros y la organización como objetos, prevalece el cinismo, la apatía, crítica de manera negativo todo el entorno laboral y el trabajo de los/las demás.

3. Baja realización personal en el trabajo: se caracteriza como una tendencia del trabajador a autoevaluarse de forma negativa, bajo rendimiento laboral, incapacidad para soportar presión, baja autoestima, insatisfacción con su desarrollo profesional y sentimiento de fracaso.

Patrick, Tak. M \& chan R (2004), definieron el Síndrome de Burnout como "un estado mental persistente y negativo que posee la persona en relación a su labor, se caracteriza por agotamiento emocional acompañado por distres, baja efectividad, poca motivación y actitudes disfuncionales en el puesto de trabajo". De manera similar Cordeiro, J, A. Guillén J, C y Gala F (2003), señalan que Burnout significa "sentirse quemado, agotado, sobrecargado o exhausto, afecta principalmente a personas cuyas tareas laborales tienen un componente central de ayuda a los demás y con gran intervención social, entre ellos la profesión docente". Con palabras más sencillas, Pando M, Aranda C, Valazquez I (2003), afirma que Burnout significa estar quemado, desgastado, exhausto y perder la ilusión por el trabajo. Lo cual explica el sentimiento de apatía y cinismo que experimentan las personas afectadas.

Jerry Edelwich y Archie Brodsky (1980), Explican las cuatro fases que atraviesa una persona antes y durante el padecimiento del Síndrome de Burnout:

1. Etapa de idealismo y entusiasmo: El individuo posee un alto nivel de energía para el trabajo, se involucra demasiado y asume tareas que no le corresponden de manera voluntaria, posee una hipervalorización de su capacidad profesional. 
2. Etapa de estancamiento: Al pasar el tiempo el individuo observa que los esfuerzos realizados en su trabajo no son recompensados, ni reconocidos, en ocasiones no logra cumplir con los compromisos adquiridos y sus expectativas se ven frustradas, esto provoca el sentimiento de desilusión. El individuo constata que sus expectativas profesionales no están siendo satisfechas, por cual, pierde el idealismo y entusiasmo hacia su trabajo.

3. Etapa de apatía: Es la fase central del síndrome: El individuo desarrolla apatía y falta de interés hacia las actividades laborales, se ausenta reiteradamente, evita el contacto con los compañeros, se ausenta del puesto laboral.

4. Etapa de distanciamiento. La persona está crónicamente frustrada en su trabajo, ocasionando sentimientos de vacío total que pueden manifestarse en la forma de distanciamiento emocional y de desvalorización profesional, en los casos más extremos dejan de ejercer la profesión.

\section{Materiales y métodos}

El estudio es cuantitativo, descriptivo y de corte transversal, realizado con docentes universitarios que laboran en las dos universidades ubicadas en la ciudad de Bluefields. De un total de 140 docentes universitarios con al menos un año de ejercer docencia universitaria, aunque estaba previsto incluir al 100\% solo se logro la participación del 78\%. Para el abordaje de los Factores Psicosociales del Trabajo a los que están expuestos los y las docentes de la ciudad de Bluefields, se utilizó la Escala de Factores Psicosociales en el Trabajo Académico, diseñado por Silva N (2006), consiste en una lista de agentes agrupados en siete subescalas. Utiliza una escala de cinco grados, tipo Likert, que va de nunca (o) a siempre (4), se suman los puntajes de cada apartado y se determinan tres niveles, baja indicando que la presencia del factor es nula o casi nula, media refleja presencia (moderada) y alta representa su existencia en gran magnitud.

La Escala de Factores Psicosociales permite analizar cada componente de manera individual utilizando los siguientes parámetros de puntuaciones:

Cuadro No. 1. Valores de la Escala Factores Psicosociales del Trabajo Academico. Silva, N (2006)

\begin{tabular}{|l|l|l|l|}
\hline \multicolumn{1}{|c|}{ Subescala } & \multicolumn{1}{c|}{ Alto } & \multicolumn{1}{c|}{ Medio } & \multicolumn{1}{c|}{ Bajo } \\
\hline Condiciones del lugar de trabajo & $27-40$ & $14-26$ & $=<13$ \\
\hline Carga de Trabajo & $17-24$ & $9-16$ & $=<8$ \\
\hline Contenido y Características de la Tarea & $22-32$ & $11-21$ & $=<10$ \\
\hline Exigencias Laborales & $19-28$ & $10-18$ & $=<9$ \\
\hline Papel Académico y Desarrollo de la carrera & $19-28$ & $10-18$ & $=<9$ \\
\hline
\end{tabular}




\begin{tabular}{|l|l|l|l|}
\hline \multicolumn{1}{|c|}{ Subescala } & \multicolumn{1}{c|}{ Alto } & \multicolumn{1}{c|}{ Medio } & \multicolumn{1}{c|}{ Bajo } \\
\hline $\begin{array}{l}\text { Interacción Social y aspectos organizacio- } \\
\text { nales }\end{array}$ & $25-36$ & $13-24$ & $=<12$ \\
\hline Remuneración y Rendimiento & $9-12$ & $5-8$ & $=<4$ \\
\hline Valores Generales & $138-200$ & $27-137$ & $=<71$ \\
\hline
\end{tabular}

Para la valoración de presencia de Burnout se utilizó Maslach Burnout Inventory, Consiste en una lista de agentes agrupados en tres dimensiones. Utiliza una escala 7 grados, tipo Likert, que va de o (nunca) a 6 (diariamente). Puntuaciones altas en las subescalas de Agotamiento Emocional y Despersonalización corresponden a altos sentimientos de estar quemado, en la subescala de realización personal las calificaciones bajas indican baja realización personal, por tanto presencia de Burnout.

Cada dimensión posee su escala de evaluación lo cual permite realizar un análisis por cada una, utilizando los siguientes parámetros.

Cuadro No. 2.: Valores del Maslach Burnout Inventory. Jackson, C. Maslach J (1981)

\begin{tabular}{|l|l|l|l|}
\hline \multicolumn{1}{|c|}{ Dimensión } & \multicolumn{1}{c|}{ Alto } & \multicolumn{1}{c|}{ Medio } & \multicolumn{1}{c|}{ Bajo } \\
\hline Agotamiento Emocional & +27 & $19-26$ & -19 \\
\hline Despersonalización & +10 & $6-9$ & -6 \\
\hline Realización Personal & +40 & $34-39$ & -30 \\
\hline
\end{tabular}

Los datos fueron tratados en el programa estadístico para Ciencias Sociales SPSS versión 17. Se calcularon medidas de frecuencia, tendencia central, Chi cuadrado y razón de prevalencia. Para el análisis de asociación entre Factores Psicosociales del Trabajo Académico y el Síndrome de Burnout, los niveles moderado y alto, fueron agrupados en una sola categoría para indicar presencia, los que puntuaron en el nivel bajo, fueron tomados como ausencia.

\section{Resultados y discusión}

Características sociodemográficas de los docentes universitarios de la ciudad de Bluefields.

El 56\% del grupo de los docentes estudiados, son del sexo Femenino y el $44 \%$ al masculino. Se encontró que el rango general de edades oscila entre 24 a 74 años. El mayor porcentaje (34\%) se concentra entre 24-35 años, la media calculada fue 42 años. Con respecto al estado civil, el 53.2\% de los participantes están casados. 
En cuanto a la distribución étnica, el grupo predominante son los mestizos con el 56.9\% (62). De los afrodescendiente el 39.4\% (43) son creoles y o.9\% garífuna (1). De los pueblos indígenas el 0.9 pertenece a la etnia misquita, el $0.9 \%$ ramas y el con el $0.9 \%$ al Ulwa, es decir, un docente indígena por etnia.

En relación a las características laborales de los y las docentes, se observa que el mayor porcentaje de docentes (30.3\%), tiene entre 4-6 años de ejercer docencia universitaria, el $33.9 \%$ realiza esta actividad en dos turnos diferentes, aunque también es interesante señalar que el $22 \%$ lo ejerce en tres turnos.

Generalmente los docentes tiempo completo brindan clases en una modalidad, pero sus funciones incluyen actividades de investigación y/o extensión comunitaria. Mientras que docentes horarios asumen varios turnos, evitando que interfiera con sus horarios laborales en las instituciones o centros de educación secundaria en donde también laboran.

En relación a los lugares de trabajo, se presenta que el 77.1\% ejercen docencia en una de las universidades, mientras que el $22.9 \%$ lo hace en ambas. Del total de docentes, $29.4 \%$ imparte al menos dos asignaturas.

\section{Factores de riesgo psicosociales}

Las puntuaciones globales de la Escala de Factores Psicosociales indican que el 90.8\% de docentes se ubican en un nivel medio, lo cual en base a las explicaciones de la Silva $\mathrm{N}$ (2006), esto indica presencia moderada de factores psicosociales negativos en el ambiente de trabajo de los docentes.

Según la distribución porcentual de la presencia de cada componente de la Escala de Factores Psicosociales del trabajo académico según la intensidad (alto, medio y bajo), se observa que el papel académico y desarrollo de la carrera se destaca en un 74.3\% sobre las demás. También se identifica que los componentes tales como condiciones del lugar de trabajo, carga laboral, contenido y características de la tarea, exigencias laborales e interacción social, también resultaron ser factores negativos pero en una etapa de alerta.

Cuadro No. 3. Presencia de Factores Psicosociales del Trabajo Académico en Docentes universitarios de Bluefields (N: 109)

\begin{tabular}{|l|l|l|l|}
\hline \multirow{2}{*}{\multicolumn{1}{|c|}{ Factores Psicosociales }} & \multicolumn{3}{c|}{ Nivel de Presencia } \\
\cline { 2 - 4 } & \multicolumn{1}{|c|}{ Alto } & \multicolumn{1}{c|}{ Medio } & Bajo \\
\hline Condiciones del lugar de trabajo & $16.5 \%$ & $80.7 \%$ & $2.8 \%$ \\
\hline Carga de Trabajo & $21.1 \%$ & $72.5 \%$ & $6.4 \%$ \\
\hline Contenido y Características de la Tarea & $9.2 \%$ & $88 \%$ & $2.8 \%$ \\
\hline
\end{tabular}




\begin{tabular}{|l|l|l|l|}
\hline \multirow{2}{*}{\multicolumn{1}{c|}{ Factores Psicosociales }} & \multicolumn{3}{c|}{ Nivel de Presencia } \\
\cline { 2 - 4 } & \multicolumn{1}{c|}{ Alto } & \multicolumn{1}{c|}{ Medio } & \multicolumn{1}{c|}{ Bajo } \\
\hline Exigencias Laborales & $39.4 \%$ & $56.9 \%$ & $3.7 \%$ \\
\hline Papel Académico y Desarrollo de la carrera & $74.3 \%$ & $25.7 \%$ & \\
\hline Interacción Social y aspectos organizacionales & & $94.5 \%$ & $5.5 \%$ \\
\hline Remuneración y Rendimiento & $26.6 \%$ & $43.1 \%$ & $30.3 \%$ \\
\hline Puntuación Global & $8.3 \%$ & $90.8 \%$ & $0.9 \%$ \\
\hline
\end{tabular}

Fuente: Elaboración propia.

$\mathrm{Al}$ igual que en este estudio, Pando. M (2006), en su investigación realizada con académicos en universidad de Guadalajara, también señala como principal factor psicosocial negativo, el papel académico y desarrollo de la carrera, esto podría estar asociado a lo que explica Silva. N (2006), al decir que los docentes, en general tiene sobrecarga mental generada por demandas cualitativas y cuantitativas específicas de este tipo de trabajo, el cual es de carácter ambivalente y contradictorio.

Llama la atención que en este estudio con docentes de Bluefields, el factor psicosocial laboral, referido a la remuneración y rendimiento no fue muy representativo, en comparación a la investigación realizada por Pando M (2006), con docentes de la universidad de Guadalajara, y por Salazar E, Martínez N, Santes C (2008), en una universidad pública del Estado de Veracruz, México, Esta similitud encontrada en estas universidades, se debe a que tradicionalmente las universidades públicas, establecen honorario más relativamente bajos en comparación a otras, si bien es cierto que las universidades del Caribe nicaragüenses, surgen como instituciones no gubernamentales, su misión y visión ha sido enfocada al servicio comunitario, sin fines de lucro, Aunque actualmente las dos universidades ubicadas en la ciudad de Bluefields, reciben un porcentaje simbólico del presupuesto nacional de Educación Superior, este "apoyo" financiero es limitado, considerando que estas universidades brindan cobertura a las dos Regiones Autónomas de Nicaragua.

De igual forma, se conoce que los honorarios que perciben los docentes universitarios por su trabajo en ambas universidades, son significativamente bajos, en comparación a otras universidades de Nicaragua, también es una realidad que en las Regiones del Caribe nicaragüense los costos de vida son elevados en comparación al resto del país, por tanto se podría decir que para los docentes universitarios de la ciudad de Bluefields, la remuneración económica, hasta el momento ha ocupado un segundo plano, siendo la prioridad y de mayor satisfacción, contribuir al desarrollo de la Región, a través de su labor en la formación de recursos humanos, Sin embargo, debe reconocerse que el factor remuneración y rendimiento lo señalan en un estado de alerta, lo cual indica, que de continuar en esta situación, llegaría a ser un factor negativo de alta incidencia, esto es consistente con los constantes cambios socioeconómicos que experimenta el país, y más aún las Regiones Autónomas. 


\section{Resultados de análisis del Maslach Burnout Inventory}

El análisis de las puntuaciones globales del Maslach Burnout Inventory, señala que académicos de la ciudad de Bluefields obtuvieron puntuaciones bajas, es decir existencia casi nula del síndrome; sin embargo, se identificaron seis docentes que padecen de este mal. Por otro lado, los resultados del Maslach Burnout Inventory por cada dimensión, muestra existencia casi nula de agotamiento emocional, presencia en un nivel medio y de alerta de la dimensión de despersonalización con el 61.5\%, indicando afectación moderada. En relación a la realización profesional, el cuadro muestra que el 56.9\% obtuvo puntuaciones altas, es decir poseen una alta realización profesional, mientras que un $30.3 \%$ se ubican en zona de alerta.

Cuadro No. 4. Prevalencia por Dimensión del Síndrome de Burnout en docentes universitarios de Bluefields (N: 109)

\begin{tabular}{|l|r|r|r|}
\hline Nivel - Dimensión & \multicolumn{1}{c|}{ Alto } & Medio & Bajo \\
\hline $\begin{array}{l}\text { Agotamiento } \\
\text { Emocional }\end{array}$ & $4.6 \%$ & $3.7 \%$ & $91.7 \%$ \\
\hline Despersonalización & $28.8 \%$ & $61.5 \%$ & $10.5 \%$ \\
\hline Realización Personal & $56.9 \%$ & $30.3 \%$ & $12.8 \%$ \\
\hline
\end{tabular}

Fuente: Elaboración propia.

Patrick L (2004), realizó un estudio con maestros y maestras de Educación Media (Hong Kong), encontrando mayores afectaciones en las dimensiones de desgaste emocional y realización personal, resultados similares obtuvieron León $S$, Matsui O, Aranda C. y colaboradores (2008) en otra investigación realizada en un centro de educación básica en Jalisco. Ambos estudios omiten información sobre la extensión del trabajo de estos docentes en el ámbito universitario, situación que podría explicar la diferencias significativas entre esos resultados y los identificados con los académicos de Bluefields, donde la presencia del componente de desgaste emocional es casi nula y una minoría presentó afectaciones en la dimensión de realización personal, Esto posiblemente esté relacionado a que ambas universidades han sido proyectos iniciados por personas autóctonas, convencidos de que la formación profesional de la población costeña es un eslabón fundamental para el progreso, hace veinte años, la mayoría de bachilleres no lograban acceder a la Educación Superior, debido a la falta de recursos económicos que les permitiera emigrar al pacifico del país o al extranjero con el fin de obtener un título profesional. En la actualidad los y las docentes han desarrollado un compromiso social intenso que les permite el cumplimiento de esta labor con orgullo y satisfacción, por tanto podría afirmarse que su desarrollo profesional está íntimamente ligado a su autorrealización personal.

A pesar del alto grado realización personal que poseen los docentes en estudio, un porcentaje significativo, presentan afectación en la dimensión de despersonalización, 
ubicándose en los niveles medio y alto, situación contraria encontraron Pando M y Castañeda J (2006), al aplicar el Maslach Burnout Inventory en una universidad de México, en ella los académicos en su mayoría se ubicaron en las puntuaciones bajas. Sin embargo, debe considerarse que la despersonalización consiste en una manifestación conductual que puede presentarse de manera sutil, evitando ser reconocida como una afectación a causa de experimentar Burnout, y ser vista, más bien, como parte de la personalidad del docente. En el caso de Bluefields, la alta presencia de Despersonalización, podría generarse a causa de las características laborales, un alto porcentaje de los y las docentes desempeñan múltiples responsabilidades laborales, lo cual conlleva a trabajar bajo constante presión y optimización del tiempo para cumplirlas con todas las demandas, lo cual conlleva a que desarrollen una respuesta automatizada y poco afectiva hacia los demás.

\section{Resultados de Factores Psicosociales del trabajo asociados a Síndrome de Burnout}

Después de realizar los análisis correspondientes entre los componentes de Factores Psicosociales negativos del trabajo con cada una de las dimensiones del Síndrome de Burnout, se encontró asociación significativa entre los siguientes componentes:

- Asociación entre el componente Contenido/características de la tarea con la dimensión de despersonalización (*P: 0.007), se identifica que docentes expuestos a contenido y características de tareas negativas reportan 2.5 veces más prevalencia de despersonalización en comparación a los que están expuestos a otros factores.

- Asociación entre el componente Papel Académico con la dimensión Agotamiento emocional (*P: 0.02), se identifica que docentes expuestos a altas demandas del Papel Académico y desarrollo de la carrera reportan 11.5 veces más prevalencia de agotamiento emocional en comparación a los expuestos a otros factores.

Cuadro No. 5. Valores de Asociación entre Presencia de Factores Psicosociales del Trabajo Académico y Síndrome de Burnout en docentes universitarios de Bluefields. (N: 109)

\begin{tabular}{|l|c|c|c|}
\hline \multirow{2}{*}{ Factores Psicosociales } & \multicolumn{3}{|c|}{ Dimensión del Burnout } \\
\cline { 2 - 4 } & $\begin{array}{c}\text { Agotamiento } \\
\text { emocional }\end{array}$ & Despersonalización & $\begin{array}{c}\text { Realización } \\
\text { profesional }\end{array}$ \\
\hline $\begin{array}{l}\text { Contenido y Características de } \\
\text { la Tarea }\end{array}$ & -- & $\begin{array}{c}\text { RP: } 2.5 \\
\text { IC (1.20-5.91) } \\
* P: 0.007\end{array}$ & -- \\
\hline $\begin{array}{l}\text { Papel Académico y Desarrollo } \\
\text { de la carrera }\end{array}$ & $\begin{array}{c}\text { RP: } 11.57 \\
\text { IC (1.35-99.27) } \\
* P: 0.02\end{array}$ & -- & - \\
\hline
\end{tabular}

*Corrección por continuidad de Yates 
Pando M y Castañeda J (2006), identificaron asociación entre las subescalas "papel académico y el desarrollo de su carrera" con las tres dimensiones del síndrome, la "carga de trabajo", "contenido y características de la tarea" mostró asociación con la dimensión de agotamiento emocional. Este estudio con docentes universitarios de la ciudad de Bluefields refleja la existencia de asociación entre el componente "papel académico y desarrollo de la carrera" con las dimensiones agotamiento emocional. Un nuevo elemento que surge es la relación entre "contenido y características de la tarea" con el ámbito de despersonalización. Esta situación puede explicarse considerando que en otros países, entre ellos México, los docentes tienen mayor claridad de su rol y funciones que deben desempeñar, en cambio en las universidades del Caribe nicaragüense los docentes realizan actividades académicas, administrativas, de investigación y extensión comunitaria. Parte de esta problemática se debe a la poca capacidad que poseen las universidades en la contratación de personal suficiente que atienda cada función, de manera que se evite la sobrecarga y diversidad en las tareas que los académicos deben realizar.

Por otro lado, la relación entre pertenecer al sexo femenino y el síndrome de Burnout también ha sido documentada. Estudios realizados Patrick L, Tak M, Chan R. (2004) con maestros de un centro de Educación Media en Hong Kong, y otro desarrollado por Pando M, Aranda Cy Aldrete M (2006) con docentes del centro universitario de ciencias de la Salud de México, identificaron que las mujeres presentan más riesgo a presentar el desgaste emocional, en cambio el estudio con docentes de Bluefields no muestra ninguna asociación entre el sexo y las dimensiones del síndrome. Ante esto, se puede mencionar que las mujeres del Caribe nicaragüense, debido a diversas experiencias vividas, han desarrollado una capacidad extraordinaria de soportar las vicisitudes de la vida (resilencia), entre ellas se pueden mencionar el residir en un país en vía de desarrollo, expuestos a la marginación, pobreza, desigualdad, inequidad, guerra, desastres naturales etc. a como describen Poseck B, Carbelo B, Jiménez B. (2006), tradicionalmente las mujeres asumen un rol integrador y de cohesión comunitaria ante los eventos mencionados, obligándolas al autocontrol emocional. Sí bien es cierto que habitantes de otros países han experimentado algunas de las condiciones aquí descritas, la historia de Nicaragua indica que estas circunstancias han sido más trágicas e impactantes en la vida de los nicaragüenses, más aún en pobladores en la Costa Caribe.

Los análisis estadísticos no mostraron asociación entre pertenecer a un grupo étnico y presentar el Síndrome, en la indagación de literatura, tampoco se encontró documentación relacionada. 


\section{CIENCIAS SOCIALES}

\section{Conclusiones}

El estudio revela que los docentes universitarios de la ciudad de Bluefields, están expuestos a factores psicosociales negativos en el trabajo, principalmente a la alta presencia del componente "papel académico y desarrollo de la carrera". Los factores relacionados con las condiciones del lugar de trabajo, carga laboral, contenido y características de la tarea, exigencias laborales e interacción social y aspectos organizacionales también resultaron ser factores negativos pero en un nivel moderado (etapa de alerta).

En cuanto al Síndrome de Burnout los docentes universitarios presentan mayores afectaciones en la dimensión de despersonalización. Sin embargo poseen un alto sentido de realización profesional.

El estudio señala asociación entre la exposición a factores psicosociales del trabajo académico y el Síndrome de Burnout, así mismo, se identifico relación entre el componente Contenido/características de la tarea con la dimensión de despersonalización.

\section{Lista de referencia}

Cordeiro, J. A., Guillén, C. I. \& Gala, F. J. (2003). Prevalencia del síndrome de Burnout en los maestros. Resultados de una investigación preliminar. Psicología.Com, 7, 1.

Freudenberger, H. J. (1974). Staff Burnout. JSoc Issues, 30:159-165.

Gil. M., \& Peiró, S. (1997). Desgaste Psíquico en el Trabajo: El Síndrome de Quemarse. $1^{\text {a }}$ edición, Madrid: Editorial

Jerry Edelwich y Archie Brodsky (1980). Burn-Off: stages of disillusionment in the helping professions. Nueva York: Human Sciences Press.

León, S., \& Matsui, O., Aranda, C., León, M., (2008). Factores Psicosociales y Síndrome de Burnout en docentes de educación básica del estado de Jalisco. Memoria 2008.

Maslach, C., \& Jackson, J. (1981). The measurement of experienced Burnout. OccupBehav,2: 99-113.

Moreno Jiménez, B., Seminotti, R., Garrosa Hernández, E., Rodríguez-Carvajal, R., Morante Benadero, M.E. (2005). El Burnout médico: la ansiedad y los procesos de afrontamiento como factores intervinientes. Ansiedad y Estrés, 11(1), 87-100.

Organización Internacional del Trabajo (2008). Enciclopedia de la Salud y Seguridad en el trabajo. Ginebra. 
Pando, M., \& Aranda, C., Aldrete, M. (2006). Factores psicosociales y Burnout en docentes del centro universitario de ciencias de la salud. MediagraficArtenisa, 8 (3)

Pando, M., \& Castañeda, J. (2006). Factores Psicosociales y Síndrome de Burnout en docentes de la universidad del Valle de Atamajac. Medigraphe (Mexico): 8(3)

Pando, M.,\& Aranda, C., Velázquez, I. (2003). Síndrome de Burnout y Factores Psicosociales en el trabajo, en estudiantes de postgrado del Departamento de Salud Pública de la Universidad de Guadalajara, México. Psiquiatría FacMed Barna, 30 (4):193-199

Patrick, L.,\& Tak, M., Chan, R. (2004). Demographic characteristic makes difference to Burnout among secondary Hong Kong teachers. Springer, 71 (11205) 004-8003.

Poseck, B., \& Carbelo, B., Jiménez, B. (2006). La experiencia traumática desde la psicología positiva: Resiliencia y crecimiento postraumático. Papeles del Psicólogo, Vol. 27(1)

Preciado, M.,\& Chávez, R. (2010). Factores Psicosociales del Trabajo e Inteligencia Emocional en la Formación Del Profesional de Enfermería de una Universidad Pública de México: Revista Colombiana de Salud Ocupacional, 1(1), 17-21

Salazar, E., \&Martínez, N., Santes, C. (2008). Factores psicosociales en la docencia que se asocian con el Sindrome de Burnout en académicos de una universidad pública del estado de Veracruz. Investigación sobre Factores Psicosociales, 56

Silva, N. (2006). Factores psicosociales, estrés y desgaste profesional en académicos del Centro Universitario de Ciencias Biológicas Agropecuarias de la Universidad de Guadalajara. Tesis de Doctorado en Ciencias de la Salud en el Trabajo de la Universidad de Guadalajara. México: Universidad de Guadalajara.

Weber, A., \&Jeakel, R. (2000). Burnout Syndrome: a disease of Modern society. Occup. Med (Great Bratain), 50 (7): 0962-7480 